\title{
Efficacy of video assisted thoracoscopic lung biopsy: an historical comparison with open lung
} biopsy

\author{
Fiona M Carnochan, William S Walker, Evan W J Cameron
}

\begin{abstract}
Background - Video assisted thoracoscopic lung biopsies were compared with historical controls undergoing open lung biopsy to determine the diagnostic accuracy, effect on length of postoperative stay, and cost effectiveness of the new thoracoscopic technique.

Methods - The first 25 video assisted thoracoscopic lung biopsies performed in the Edinburgh Thoracic Unit were compared with 25 historical controls for complications, diagnostic accuracy, and length of postoperative stay.

Results - Statistical comparison showed equal diagnostic accuracy in both groups (96\% v 92\%), but mean (SD) inpatient stay was reduced in the video assisted thoracoscopic group (1.4 (0.7) days) compared with those undergoing open lung biopsy (3.1 (1.8) days). No postoperative complications were reported in the group which underwent video assisted thoracoscopic lung biopsies but three patients had postoperative complications in the open lung biopsy group.

Conclusions - Video assisted thoracoscopic lung biopsy is as effective in providing histological diagnosis as is open lung biopsy. All postoperative complications were related to post thoracotomy pain and occurred only in patients undergoing open lung biopsy. Reduced postoperative disability in the video assisted thoracoscopic group decreased hospital stay, offsetting the increased cost in disposables. The overall cost of video assisted thoracoscopic and open lung biopsy was $£ 712$ and $£ 1114$, respectively.
\end{abstract}

(Thorax 1994;49:361-363)

The desire to obtain a definitive diagnosis before commencing potent medical treatments with drugs such as corticosteroids or immunosuppressive drugs ${ }^{1-3}$ has increased the demand for lung biopsy. Video assisted thoracoscopic techniques allow operative access to the pleural cavity without recourse to thoracotomy, a procedure not without significant morbidity. ${ }^{45}$ The technique of video assisted thoracoscopic lung biopsy appears to reduce postoperative pain and functional disability, allowing earlier discharge. However, the video assisted technique is only an alternative method of achieving an established surgical goal and must therefore be shown to be as effective as the open procedure. Additionally, video assisted thoracoscopic surgery incurs added financial costs in the use of disposable items. We present our experience with video assisted thoracoscopic lung biopsy and open lung biopsy to determine the efficiency, cost, and quality outcome of the new method.

\section{Methods}

Between March and September 1992 the first 25 patients underwent video assisted thoracoscopic lung biopsy in our unit and, for the purpose of comparison, the results of 25 patients undergoing open lung biopsy, as closely matched as possible for age and sex, were selected from the previous two years. Diagnosis, complications, duration of hospital stay, and nature of discharge were determined retrospectively. The average cost of hospital stay was derived from hospital administration data, and the cost of surgical disposable items was derived from current contract prices.

The requirement for convalescence before hospital discharge was also studied. On review of a patient's medical records convalescence was considered necessary if the patient had postoperative pain requiring opiates, or the patient had a significant postoperative chest infection.

\section{SURGICAL TECHNIQUE}

Both open lung biopsies and video assisted thoracoscopic lung biopsies were performed under general anaesthesia with the patient in the lateral decubitus position. Anaesthetic technique was identical in both groups. All patients had double lumen endotracheal tubes placed and received intercostal nerve blocks with $0.5 \%$ bupivacaine and adrenaline before surgical intervention. Anaesthetic agents chosen were not dependent on the biopsy method.

\section{Open lung biopsy}

A 4 inch "mini" thoracotomy was made in the mid lateral chest to divide the underlying portion of the latissimus dorsi muscle, but sparing the serratus anterior muscle. An intercostal incision was made and the ribs separated with a Touffier retractor. A suitable portion of lung was identified, excluded with a linear gastric stapler (TA55; Autosuture, UK), and excised. The wound was then closed with pericostal and layered Vicryl and a single inferior intercostal drain was inserted. 
Table 1 Details of patients undergoing open lung biopsy and video assisted thoracoscopic lung biopsy

\begin{tabular}{|c|c|c|c|c|}
\hline \multirow[t]{2}{*}{ Patient no. } & \multicolumn{2}{|c|}{ Open lung biopsies } & \multicolumn{2}{|c|}{ Video assisted thoracoscopic lung biopsies } \\
\hline & Age & Postoperative stay (days) & Age & Postoperative stay (days) \\
\hline 1 & 55 & 2 & 53 & 2 \\
\hline 2 & 52 & 4 & 51 & 1 \\
\hline 3 & 57 & 1 & 53 & $1(T)$ \\
\hline 4 & 72 & $3(\mathrm{~T})$ & 71 & 2 \\
\hline 5 & 64 & 4 & 74 & 1 \\
\hline 6 & 50 & 8 & 49 & 1 \\
\hline 7 & 51 & 2 & 50 & 1 \\
\hline 8 & 71 & 1 & 71 & 3 \\
\hline 9 & 34 & 1 & 34 & 1 \\
\hline 10 & 62 & 4 & 63 & $8^{*}(3)$ \\
\hline 11 & 46 & 5 & 41 & 3 \\
\hline 12 & 55 & 2 & 46 & 1 \\
\hline 13 & 80 & $3(\mathrm{~T})$ & 81 & 1 \\
\hline 14 & 45 & 2 & 39 & 1 \\
\hline 15 & 74 & 2 & 72 & 2 \\
\hline 16 & 42 & 6 & 37 & 1 \\
\hline 17 & 66 & $3(\mathrm{~T})$ & 66 & 2 \\
\hline 18 & 60 & 3 & 66 & 1 \\
\hline 19 & 64 & $6(T)$ & 63 & 1 \\
\hline 20 & 63 & $3(\mathrm{~T})$ & 60 & 2 \\
\hline 21 & 57 & 2 & 51 & 1 \\
\hline 22 & 64 & 4 & 68 & 1 \\
\hline 23 & 65 & 2 & 65 & 1 \\
\hline 24 & 64 & 1 & 74 & 1 \\
\hline 25 & 33 & $4(T)$ & 40 & 1 \\
\hline
\end{tabular}

$\mathrm{T}=$ patient transferred for further convalescence to referral hospital.

* Patient fit for discharge after three days but remained in the unit because of transport difficulties.
Video assisted thoracoscopic lung biopsy

This was performed with three $1 \mathrm{~cm}$ stab incisions in the lateral chest wall. A $10 \mathrm{~mm}$ laparoscope was introduced through the most posterior of these incisions. An Endo-GIA 30 stapler and Rampley's sponge holding forceps (used for grasping lung) were sited in the other two ports. After taking the biopsy material two of the wounds were closed with Vicryl and the third used for an intercostal drain.

Patients returned to the ward and the intercostal drain was removed either the same evening or the next day when there was no air leak and the lung was fully expanded. Postoperative care was identical in both groups.

\section{STATISTICAL ANALYSIS}

Statistical analysis of the data was performed using the paired Student's $t$ test.

\section{Results}

The group which underwent open lung biopsy consisted of 11 women and 14 men of mean (SD) age $57.8(11.8)$ years (range $33-80$ ); the video assisted thoracoscopic group included 10 women and 15 men of mean (SD) age 57.5

Table 2 Complications, diagnostic accuracy, length of postoperative stay and convalescence in patients undergoing open lung biopsy and video assisted thoracoscopic lung biopsy

\begin{tabular}{lll}
\hline & $\begin{array}{l}\text { Open lung } \\
\text { biopsy } \\
(n=25)\end{array}$ & $\begin{array}{l}\text { Video assisted thoracoscopic } \\
\text { lung biopsy } \\
(n=25)\end{array}$ \\
\hline Mean (SD) age (range) & $57 \cdot 8(11 \cdot 8)$ & $\begin{array}{l}57 \cdot 5(13 \cdot 4) \\
34-81\end{array}$ \\
No. of patients with & $33-80$ & \\
postoperative complications & 3 & 0 \\
$\begin{array}{l}\text { Definitive diagnosis } \\
\text { Mean (SD) postoperative stay (range) in days }\end{array}$ & 23 & 24 \\
& $1-8$ & $1 \cdot 4(0 \cdot 7)$ \\
$\begin{array}{l}\text { Number of patients transferred for further } \\
\text { convalescence }\end{array}$ & 6 & $<24$ hours-8* \\
\hline
\end{tabular}

* Transport difficulties prolonged stay.
(13.4) years (range 34-81). Details of the age and length of postoperative stay (including the time in the thoracic unit following the day of surgery) are shown in table 1 . Complications, diagnostic accuracy, length of postoperative stay, and convalescence are summarised in table 2 . The mean length of postoperative stay for the open lung biopsy and video assisted thoracoscopic groups was 3.1 and 1.4 days, respectively $(\mathrm{p}<0.0001)$. No patients in the video assisted thoracoscopic group had postoperative complications, whereas three patients undergoing open lung biopsy had postoperative complications all related to pain. One patient required referral to the pain clinic for continued thoracotomy pain months after the initial procedure, another patient reattended the thoracic unit for intercostal nerve blocks, and the third suffered a chest infection with lower lobe collapse postoperatively as a result of reduced respiratory effort secondary to postoperative pain. Six patients undergoing open lung biopsy had to be transferred for additional convalescence, but only one patient in the video assisted thoracoscopic group required further convalescence.

\section{Discussion}

This study confirms the feasibility of video assisted thoracoscopic lung biopsy and shows that it is at least as effective as open lung biopsy in obtaining a histological diagnosis. All postoperative complications were related to post thoracotomy pain and were seen only in patients undergoing open lung biopsy. Reduced postoperative disability in the video assisted thoracoscopic group shortened postoperative stay, offsetting the increased cost of disposables; $£ 316$ in the video assisted thoracoscopic group and $£ 231$ in the open lung biopsy group. Taking account of the cost of a routine hospital bed, the overall costs of video assisted thoracoscopic and open lung biopsy procedures were $£ 712$ and $£ 1114$ respectively, making an overall saving of $£ 402$ for video assisted thoracoscopic cases. These costs only take into account hospital stay and disposable costs, but further savings may be made in the video assisted thoracoscopic group with a reduced need for convalescence and treatment of post thoracotomy pain.

The technique of video assisted thoracoscopic lung biopsy is more efficient as it saves operative time and provides good visualisation of the lung and pleural surfaces. In cases where the extent of the lung disease is limited it allows the operator to remove biopsy specimens from sites which are macroscopically abnormal. Mini-thoracotomy, on the other hand, provides only limited visualisation and the biopsy material is usually taken from the area most easily accessed.

In some cases video assisted thoracoscopic lung biopsy is not possible as extensive pleural adhesions prevent placement of the camera into the pleural cavity, and an open lung biopsy is then necessary.

During this retrospective study it was necessary to obtain matched historical open biopsy 
controls over a two year period before March 1992. Video assisted thoracoscopic cases were collected consecutively between March and September 1992. The need to collect open lung biopsy controls over a considerably longer period followed a change in referral pattern for lung biopsy. Previously the demand for lung biopsy was relatively small, but since the advent of the video assisted thoracoscopic technique there has been a rise in the numbers of referrals for lung biopsy. This change may reflect a change in attitude among physicians towards commencing potent medical treatments without a definitive histological diagnosis, or a greater acceptance of a procedure which they may feel is less invasive than open lung biopsy.

Video assisted thoracoscopic lung biopsy is a cost effective and more acceptable alternative to open lung biopsy which can provide an accurate diagnosis in the management of pulmonary infiltrates.

1 Turner-Warwick RH, Burrows B, Johnson A. Cryptogenic fibrosing alveolitis. Response to corticosteroid treatment and its effect on survival. Thorax 1980;35:593-9.

2 Winterbauer RH, Hammar SP, Hallman KO, Hay JE, Neeley E, Morgan EH, et al. Diffuse interstitial pneumonitis: clinicopathological correlations in 20 patients treated tis: clinicopathological correlations in 20 patients treated
with prednisolone/azathioprine. $A m \mathcal{M}$ (1978;65:66172 .

3 Hazelrigg SR, Boley TM, Schmaltz RA, Johnson JA, Curtis $\mathrm{JJ}$. The effect of muscle-sparing versus standard posterolateral thoracotomy on pulmonary function, muscle strength and postoperative pain. $\mathcal{F}$ Thorac Cardiovasc Surg 1991;101:394-401.

4 Jayr C, Mollie A, Bourgain JL, Alarcon J, Masselot J, Lasser $\mathrm{P}$, et al. Postoperative pulmonary complications: general anaesthesia with postoperative parenteral morphine com-
pound with epidural analgesia. Surgery 1988;104:B57-63.

5 Bateman ED, Turner-Warwick M, Haslam PI, AdelmannGrill BC. Cryptogenic fibrosing alveolitis: prediction of fibrogenic activity from immunohistochemical studies of collagen types in lung biopsy specimens. Thorax 1983;38:93-101. 